\title{
Influence of the surface roughness and oxide surface layer onto Si optical constants measured by the ellipsometry technique
}

\author{
T.S. Rozouvan, L.V. Poperenko, I.A. Shaykevich \\ Taras Shevchenko Kyiv National University, Department of Physics, \\ 4, prospect Glushkova, 03187 Kyiv, Ukraine \\ E-mail:tamara_rozouvan@yahoo.ca
}

\begin{abstract}
Si crystal surface after chemical etching was studied using ellipsometry, atomic force microscopy and scanning tunneling microscopy. The ellipsometric parameters as functions of light incidence angles at two light wavelengths 546.1 and $296.7 \mathrm{~nm}$ were measured. The calculations based on equations for the plane surface have shown that the refractive index and absorption coefficient values are different from those determined earlier. Two models for surface layers were developed. After etching, the upper layer contains chemical compounds and the lower layer characterizes the sample roughness. By applying Airy's formula to ellipsometric data, optical constants and thicknesses of the layers were obtained. The calculated values of bulk Si optical constants wholly correspond to the data from literature. The calculated thickness of the lower layer is similar to that obtained through scanning tunneling microscopy measurements. Calculations based on Maxwell-Garnett and Bruggeman equations were performed to determine the content of silicon particles within the lower rough layer.
\end{abstract}

Keywords: Si nanoislands, scanning tunneling microscopy, spectral ellipsometry.

Manuscript received 12.08.14; revised version received 02.12.14; accepted for publication 19.02.15; published online 26.02.15.

\section{Introduction}

Ellipsometric experimental techniques are most commonly used for measurements of such optical constants as refractive and absorption indices inherent to metals or semiconductors within a strong absorption spectral band $[1,2]$. It's also known that the equations used to calculate optical constants based on experimentally obtained ellipsometric data are obtained for geometrically ideal plane surface of the sample. At the same time, the polished surface of the samples is rough, and there is a covering layer of oxide and other molecules [2,3]. The latter influences the ellipsometric studies and respective values of the calculated optical constants. The problem of simultaneous consideration of the influence of surface roughness and the interface layer influence on the results of ellipsometric measurements is quite complex. Most of researches in the following works either determines the latter factor [3-7] or the former one [3, 8-12]. While we managed to simultaneously establish the influence of the interface layer and the roughness on the optical constants of $\mathrm{Si}$ sample obtained by ellipsometry experiments.

\section{Experimental and results}

In our experiments, the chosen $n$-type phosphorus-doped silicon substrate with the surface plane [111] was similar to those used in solar batteries. It was etched in chemical solution based on the mixture of $\mathrm{HNO}_{3}-\mathrm{HF}, \mathrm{Na}_{2} \mathrm{Cr}_{2} \mathrm{O}_{7}$, 
$\mathrm{HNO}_{3}$ and $\mathrm{HF}$ in order to make the sample surface smoother. These chemical compounds are usually used to polish Si plates, although in our case it diminished roughness of our sample surface.

The sample surface studies were performed using the INTEGRA NT-MDT atomic force microscope that allows to perform both atomic force and scanning tunneling microscopy measurements. Ellipsometric experiments were made using the Beattie technique [2]. Parameters, such as the amplitude component $\psi$ and the phase difference $\Delta$, were measured for various angles of incidence at two wavelengths 546.1 and $296.7 \mathrm{~nm}$. These wavelengths were chosen within the spectral region of strong interband transitions for Si. The ellipsometric measurements are most effective in these spectral regions. Being based on the obtained data, the sample optical constants - refractive index $n$ and absorption index $k$-were calculated.

The well-known theoretical relations for these calculations $[1,2]$ were applied. In order to determine the roughness and the interface oxide layer influences on the final results, the sample was modeled as that having two layers on its surface (Fig. 1).

A light beam propagates from air $l(n=1)$ and then through the oxide layer 2, roughness layer 3 and finally reaches the bulk Si plate 4 . Multiple beam interference occurs in these layers, which can be described by applying Airy's formula [13]. The layer 3 is structured with Si surface irregularities (shaded in Fig. 1) and filled gaps by upper layer 2 substance. The layer 3 is considered in the model as the homogenous planeparallel one with an effective refractive index $n_{3}$.

The thicknesses of layers and their refractive indices were calculated using the measured dependences $\tan (\psi)$ and $\cos (\Delta)$ on the angle of incidence $f$, applying Eq. (1). For the layer 3, we obtain an effective refractive index. All the obtained values for refractive indices are complex $n+i k$ :

$R_{s, p_{i}}=\frac{r_{s, p_{i}}+R_{s, p_{i+1}} e^{2 i g_{i}}}{1+r_{s, p_{i}} R_{s, p_{i+1}} e^{2 i g_{i}}}$,

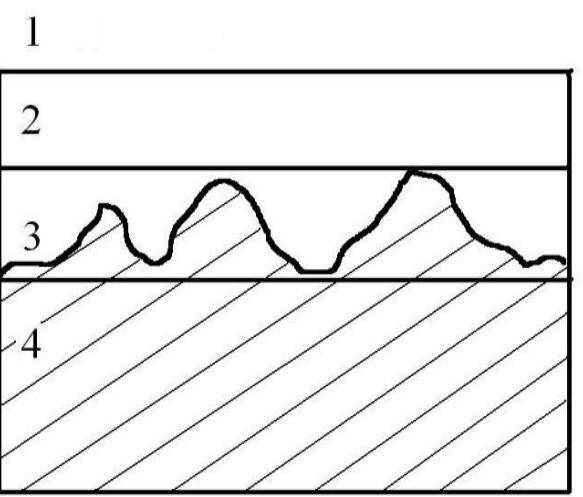

Fig. 1. Schematic cross-section of the sample. Air (1) and oxide (2), interface layers of roughness (3) on the sample surface (4). here $i$-indices mark the layers (reflection between $i$ and $i+1$ layers) $r_{s, p_{i}}$, in Eq. (1) - amplitude Fresnel reflection coefficients related to the interface between $i$ and $i+1$ layers:

$r_{s_{i}}=\frac{n_{i} \cos \left(f_{i}\right)-n_{i+1} \cos \left(f_{i+1}\right)}{n_{i} \cos \left(f_{i}\right)+n_{i+1} \cos \left(f_{i+1}\right)}$,
$r_{p_{i}}=\frac{n_{i+1} \cos \left(f_{i}\right)-n_{i} \cos \left(f_{i+1}\right)}{n_{i+1} \cos \left(f_{i}\right)+n_{i} \cos \left(f_{i+1}\right)}$,

$n_{i}$ and $f_{i}$ are the complex refractive index and light incidence angle, respectively. $g_{i}-i$ layer phase thickness taken as $g_{i}=\frac{2 \pi}{\lambda} n_{i} d_{i} \cos \left(f_{i}\right)$, where $d_{i}$ is the $i$ layer thickness and $\lambda$ - light wavelength. $R_{s, p_{i}}$ - amplitude reflection coefficient from the previous layer, calculated similarly using Eq. (1), or equal to the Fresnel coefficient for the last layer 4. Finally, we calculated the rest of the parameters from the equation

$R_{p 1} / R_{s 1}=\tan (\psi) \exp (\Delta)$

checking the fit between the experimental and theoretical data.

\section{Discussion}

The surface of our Si sample was first studied by the atomic force microscopy technique. The results of the experiments are presented in Fig. 2. As one can see, the surface is covered by irregular pyramid-shaped parts with the micron order of magnitude difference in their height and size. The faces of the pyramidal parts are not parallel to the sample surface plane, so we were not able to perform the ellipsometric experiments with such a surface. Therefore, the sample surface was etched, which resulted in a radical smoothing of the surface. The surface profile after etching is presented in Figs. 3 and 4. The data was obtained using atomic force microscopy (Fig. 3) and high resolution scanning tunneling microscopy (Fig. 4). We can see in Fig. 3 two important features of the scanned surface. First of all, the pyramidal structure from Fig. 2 is absent as a result of etching. The surface is flat, though some particles, which can be remnants of chemical reactions, are present on the surface. Vertical lines in the figure are "shadow" artifacts of the measurements because of finite cantilever needle width and sharp steps in height on the sample surface. Second, one can see straight lines a few microns in length on the surface either as the edges of a flat structure (left side of the picture) or just a dark straight line (right side of the picture). The structures may reflect crystal lattice patterns on the surface, which were studied afterward applying high resolution scanning tunneling microscopy (Fig. 4). Fig. 4 surface profile is covered by nano-islands with differences in height $10 \mathrm{~nm}$ order of magnitude, which makes the ellipsometry measurements possible. The sides of the 
nanoislands are ordered forming straight lines. The ellipsometric measurements results - dependences $\tan (\psi)$ and $\cos (\Delta)$ depending on incidence angle $f$ at the wavelength $\lambda=546.1 \mathrm{~nm}-$ are presented in Figs. 5 and 6. Similar measurements for the wavelength $\lambda=$ $296.7 \mathrm{~nm}$ are also presented in Figs. 7 and 8.

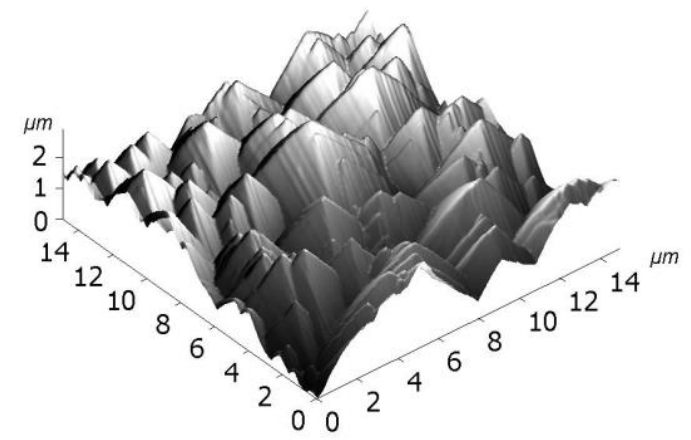

Fig. 2. Surface of the sample before etching in accord with the data of atomic force microscopy.

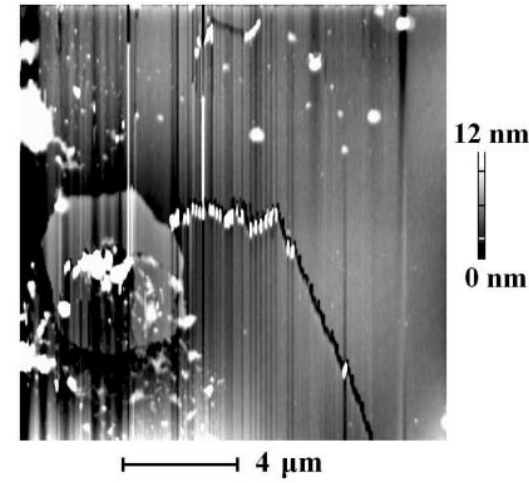

Fig. 3. Atomic force microscopy scans for the sample after etching.
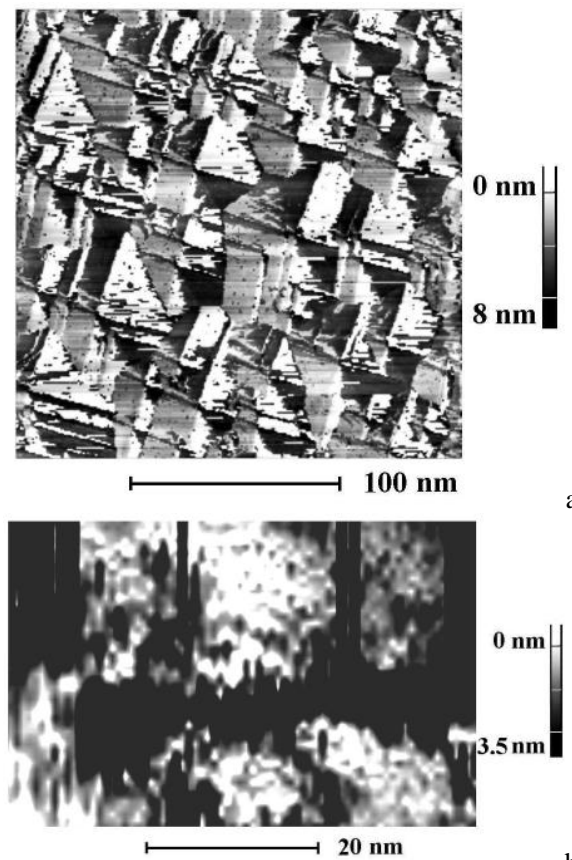

)

b)

Fig. 4. Scanning microscopy images for the sample after etching. Spatial resolution $(b)$ is five times higher than in $(a)$.
As one can see from Figs. 5 and 6, applying the equations (1)-(3) we calculated $n_{2}, k_{2}, n_{3}, k_{3}$, as well as film thicknesses $d_{2}$ and $d_{3}$. The values of $n_{4}$ and $k_{4}$ for $\mathrm{Si}$ substrate at the light wavelength $546.1 \mathrm{~nm}$ were taken from the known source [14]. The values used by us were $n_{4}=4.97$ and $k_{4}=0.044$. A similar procedure was made with calculations at $296.7 \mathrm{~nm}$ (based on the results presented in Figs. 7 and 8). Because of transcendental character of Eqs. (1) and (2), we were only able to perform numerical calculations at PC by minimizing the difference between the experimental data and numerical simulation results.

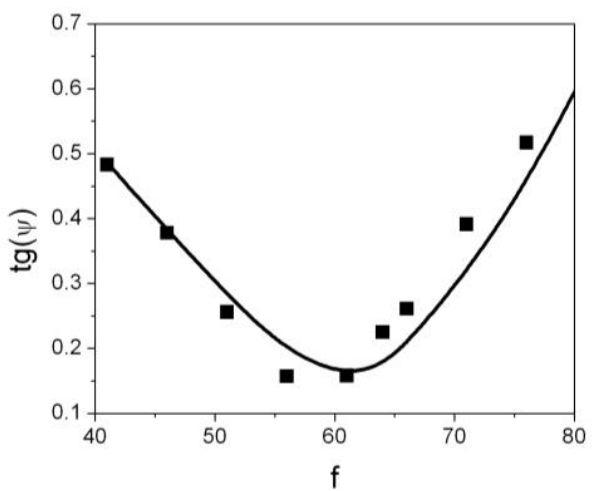

Fig. 5. Ellipsometric data: $\tan (\psi)$ as a function of the angle of incidence $f$. The etched sample at the light wavelength $546.1 \mathrm{~nm}$. Squares - experimental data, solid line - results of calculations.

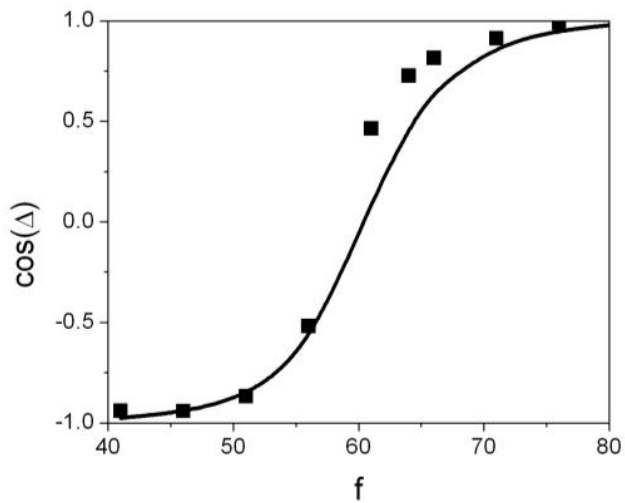

Fig. 6. Ellipsometric data: $\cos (\Delta)$ as a function of the angle of incidence $f$. The etched sample at the light wavelength $546.1 \mathrm{~nm}$. Squares - experimental data, solid line - results of calculations.

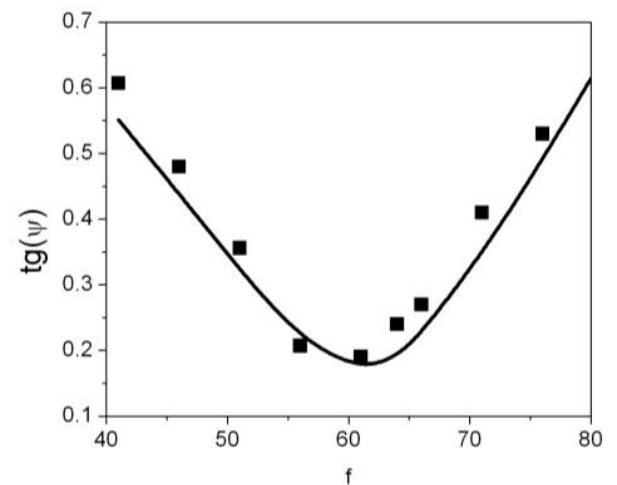

Fig. 7. Ellipsometric data: $\tan (\psi)$ as a function of the angle of incidence $f$. The etched sample at the light wavelength $296.7 \mathrm{~nm}$. Squares - experimental data, solid line - results of calculations.

\section{(C) 2015, V. Lashkaryov Institute of Semiconductor Physics, National Academy of Sciences of Ukraine}




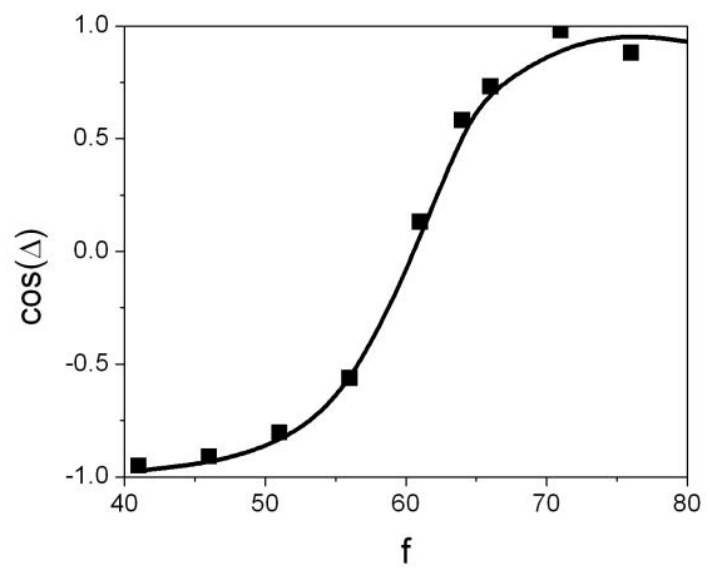

Fig. 8. Ellipsometric data: $\cos (\Delta)$ as a function of the angle of incidence $f$. The etched sample at the light wavelength $296.7 \mathrm{~nm}$ Squares - experimental data, solid line - results of calculations.

The conducted numerical simulations brought the following outcome, by applying basic equations for optical constants [1, 2], without taking into account surface interface layers,

$$
\begin{aligned}
& a=\sin (f) \tan (f) \frac{\cos (2 \psi)}{1+\sin (2 \psi) \cos (\Delta)}, \\
& b=\sin (f) \tan (f) \frac{\sin (2 \psi) \sin (\Delta)}{1+\sin (2 \psi) \cos (\Delta)}, \\
& n^{2}-k^{2}=a^{2}-b^{2}+(\sin (f))^{2}, \\
& n k=a b,
\end{aligned}
$$

we obtained $n_{4}=1.61$ and $k_{4}=0.331$ at the light wavelength $546.1 \mathrm{~nm}$. These values differ from those taken from [14]. The more drastic difference between our numerical simulations and data of [14] take place at the light wavelength $296.7 \mathrm{~nm}$. The exact values taken from [14] are $n_{4}=4.94, k_{4}=4.48$ and are clearly out of the number range of the calculations by using (4) with the values $n_{4}=1.38, k_{4}=0.344$. The results can be explained by taking into account shorter light wavelength of the reflected light with having more influence on the parameters of the sample surface. The calculations using Eqs. (1)-(3) and Figs. 5-8 data resulted in the following plots (Fig. 7):

$\lambda=546.1 \mathrm{~nm}, n_{2}=2.8, k_{2}=0.3, n_{3}=3.8, k_{3}=0.1, d_{2}=$ $50 \mathrm{~nm}, d_{3}=4.5 \mathrm{~nm}, n_{4}=4.97, k_{4}=0.044$;

$\lambda=296.7 \mathrm{~nm}, n_{2}=2, k_{2}=0.384, n_{3}=4.7, k_{3}=1.2, d_{2}=$ $50 \mathrm{~nm}, d_{3}=4.5 \mathrm{~nm}, n_{4}=4.94, k_{4}=4.48$.

By analyzing the adduced results, we can conclude:

1. The obtained data for the substrate 4 are the same with those taken from [14].

2. The thickness of the upper layer 2 containing oxides and chemical compounds as a result of $\mathrm{Si}$ substrate etching and the roughness layer 3 thickness are the same for the both light wavelengths.
3. The thickness of the roughness layer 3 is consistent with Fig. 4 data. The thickness does not exceed $10 \mathrm{~nm}$.

4. Optical constants of the roughness layer 3 differ from the constants of the chemical compounds in the lower layer, which is the result of the etching procedure, and the values are close to those of bulk $\mathrm{Si}$. The latter is pretty understandable because the layer 3 contains particles of pure Si (Fig. 1).

The point 4 allows us to apply the Maxwell-Garnett model [15] to determine the percentage of Si particles in the whole layer 3 volume using the equation:

$$
\left(\varepsilon_{3}-\varepsilon_{2}\right) /\left(\varepsilon_{3}+2 \varepsilon_{2}\right)=q\left(\varepsilon_{4}-\varepsilon_{2}\right) /\left(\varepsilon_{4}+2 \varepsilon_{2}\right),
$$

where $q=V_{\mathrm{Si}} / V, V_{\mathrm{Si}}$ is the $\mathrm{Si}$ volume in the layer $3, V-$ layer 3 volume; $\varepsilon=n^{2}$ for all layers.

The results of calculations: $q=0.687$ for $\lambda=$ $296.7 \mathrm{~nm}, q=0.527$ for $\lambda=546.1 \mathrm{~nm}$.

The $q$-numbers divergence at different light wavelengths is the result of the pyramidal shape inherent to the particles in the layer instead of the spherical shape required by Maxwell-Garnett model. The Bruggeman relation was applied to further analyze experimental data because calculated $q$ exceeds 0.5 [3]:

$q=1-\left(\varepsilon_{3}-\varepsilon_{4}\right)\left(\varepsilon_{2}+2 \varepsilon_{4}\right) /\left(\varepsilon_{2}-\varepsilon_{4}\right)\left(\varepsilon_{3}+2 \varepsilon_{4}\right)$.

The results of calculations by using Eq. (6) are as follows: $q=0.583$ for $\lambda=296.7 \mathrm{~nm}$ and $q=0.46$ for $\lambda=$ $546.1 \mathrm{~nm}$. The comparison between calculations with Eq. (5) and Eq. (6) results in lower numbers for the Bruggeman approach. The explanation is similar to the above presented and is based on a non-spherical shape of the particles.

\section{Conclusions}

1. Our studies show that the calculations based on ellipsometric data without taking into account surface interface layers of the $\mathrm{Si}$ crystal with the etched surface produce the values of optical constants different from those previously found for crystalline $\mathrm{Si}$.

2. We used Airy's formula to experimental data while applying the model of two-layered surface with the upper layer that stems from the etching procedure and the bottom roughness layer. The calculated values of optical constants are similar to those found in literature.

3. The bottom layer thickness calculated from the ellipsometric data characterizes $\mathrm{Si}$ sample roughness and is equal to $4.5 \mathrm{~nm}$, which is in agreement with the results obtained with scanning tunneling microscopy.

4. Using the Maxwell-Garnett and Bruggeman models, we calculated the partial ratio of $\mathrm{Si}$ particles in the layer, which characterizes the surface roughness. The calculations were based on the obtained optical constants for two upper layers. 


\section{References}

1. A.V. Sokolov, Optical Properties of Metals. GIFML, Moscow, 1961 (in Russian).

2. M.M. Noskov, Optical and Magneto-optical Properties of Metals. Fizmatlit, Sverdlovsk, 1983 (in Russian).

3. L.V. Poperenko, V.S. Stashchuk, I.A. Shaykevich, V.A. Odarych. Surface Diagnostic by Polarized Light. Kyiv University Publ., Kyiv, 2007 (in Ukrainian).

4. I.N. Shkliarevsky, V.P. Kostiuk, V.R. Karas, Surface films influence on magneto-optical measurements data // Optika i spectroskopiya, 23(1), p. 147-152 (1967), in Russian.

5. R.W. Collins, I. An, H.V. Nguyen, Y. Lu, Real time spectroscopic ellipsometry for characterization of nucleation, growth and optical functions of thin films // Thin Solid Films, 233, p. 244-247 (1993).

6. M. Fried, H. Wormeester, E. Zoethoud, T. Lohner, O. Polgar, I. Barsony, In situ spectroscopic ellipsometric investigation of vacuum annealed and oxidized porous silicon layers // Thin Solid Films, 459, p. 313-314 (1998).

7. W. Lehnert, P. Petric, C. Schneider, L. Pfitzner, H. Ryssel, In situ layer characterization by spectroscopic ellipsometry at high temperatures // ULSI Conference at NIST (1998).
8. V.A. Antonov, V.I. Pshenitsyn, Polaryzed light reflection by rough surface // Optika $i$ spektroscopiya, 56(1), p. 146-154 (1984), in Russian.

9. A.V. Rzhanov, S.N. Svitasheva, K.K. Svitashev, Determination of ellipsometric data of rough surfaces by graphical technique // DAN USSR, 273(5), p. 1123-1126 (1983), in Russian.

10. S. Logothetidis, Surface-roughness and grainboundary effects on the optical properties of lowpressure chemical-vapour-deposited silicon thin films by spectroscopic ellipsometry // J. Appl. Phys. 65, p. 2416 (1989).

11. S.J. Fang, W. Chen, T. Yamanaka, C.R Helms, Comparison of $\mathrm{Si}$ surface roughness measured by atomic force microscopy and ellipsometry // Appl. Phys. Lett. 68, p. 2837 (1996).

12. C. Flueraru, N. Gartner, C. Rotaru, D. Dascalu, G. Andriescu and P. Cosmin, Spectro-ellipsometric investigation of polycrystalline silicon surface roughness // Microelectron. Eng. 31, p. 309 (1996).

13. M. Born and E. Wolf, Principles of Optics. Nauka, Moscow, 1970 (in Russian).

14. E.D. Palik, Handbook of Optical Constants of Solids. Academic Press London, Boston, New York, 1998.

15. Z.G. Meyksin, Non-solid and Cermet Films in Thin Films Physics. Mir, Moscow, 8, p. 106-179 (1978), in Russian. 